\title{
Mathematical Modeling and Intelligent Algorithm for Multirobot Path Planning
}

\author{
Jason Gu, ${ }^{1}$ Xin $\mathrm{Ma}^{2}{ }^{2}$ Fei Liu, ${ }^{3}$ Ying Wang, ${ }^{4}$ and Hongliang Ren ${ }^{5}$ \\ ${ }^{1}$ Dalhousie University, Halifax, NS, Canada \\ ${ }^{2}$ Shandong University, Shandong, China \\ ${ }^{3}$ LaTrobe University, Melbourne, VIC, Australia \\ ${ }^{4}$ Kennesaw State University, Marietta, GA, USA \\ ${ }^{5}$ National University of Singapore, Singapore \\ Correspondence should be addressed to Jason Gu; jason.gu@dal.ca
}

Received 30 October 2016; Accepted 30 October 2016; Published 4 January 2017

Copyright (c) 2017 Jason Gu et al. This is an open access article distributed under the Creative Commons Attribution License, which permits unrestricted use, distribution, and reproduction in any medium, provided the original work is properly cited.

In recent years, multirobot systems have attracted many researchers' attention owing to their important applications in industry, agriculture, human life, and so forth. Path planning with obstacle avoidance is one of the most fundamental functions for mobile robots. Path planning that serves as the basis for the wheeled robot, unmanned aerial vehicle (UAV), cleaning robot, automated harvesters, rescue robot, and so forth includes formation path planning and coverage path planning. Finding an optimal path that achieves tasks is NP-hard problem. In order to find the optimal path, robots need to be equipped with sophisticated task planners that employ symbolic reasoning to move in dynamic environments. Intelligent algorithms used for path planning have not been understood completely.

In order to adjust the robot's controller, a mathematical modeling with kinematic and dynamic analysis of a mobile robot also is needed. A functional model of robot, which is created afterwards, has a better maneuverability and stability to accomplish different tasks in different environments. Quantitative measures and calculations of the mathematical modeling and intelligent algorithms are required in multirobot path planning.

This special issue is focused on the mobile robot path planning with different algorithms. This special issue has received a total of 21 submitted papers with only 5 papers accepted.

In paper "Path Planning for Rapid Large-Angle Maneuver of Satellites Based on the Gauss Pseudospectral Method,"
W. Zhang et al. have developed an attitude maneuver optimization method for gimbal rates based on the Gauss pseudospectral method. It satisfies the control requirement of rapid maneuver for satellite and evades the singularity problem of SGCMGs. This method has higher solution precision and a fast convergence rate, and it avoids the difficulties in solving a boundary value problem. According to the definition of rapid maneuvering where the attitude rate of a satellite is $1 \sim 10^{\circ} / \mathrm{s}$, the planning angular speed is $5.7^{\circ} / \mathrm{s}$. Then, through mathematic simulations based on MAT$\mathrm{LAB} /$ Simulink, the errors of quaternion and angular speed are obtained within $4 \times 10^{-4}$ and $10^{-3} \mathrm{rad} / \mathrm{s}$. In conclusion, the Gauss pseudospectral method can effectively plan an optimal trajectory, satisfying all constraints within a short time.

In paper "Vehicle Sideslip Angle Estimation Based on General Regression Neural Network," W. Wei et al. have proposed a estimation method of slip angle based on general regression neural network (GRNN) and driver-vehicle closed loop system. Vehicle's sideslip angle has been treated as time series mapping of yaw speed and lateral acceleration. The paper uses homogeneous design approach to optimize the training samples. The mapping relationship among sideslip angle, yaw speed, and lateral acceleration has been built. At the same time, vehicle's sideslip angle has been measured to verify the proposed method. Estimation results of neural network and real vehicle experiment show the same changing tendency. The mean of error is within $10 \%$ of test result's 
amplitude. Results show that GRNN can estimate vehicle's sideslip angle correctly. It can offer a reference to the application of vehicle's stability control system on vehicle's state estimation.

In paper "The Robot Path Planning Based on Improved Artificial Fish Swarm Algorithm," Y. Zhang et al. have introduced an attenuation function $\alpha$ to improve the weak point of visual in standard AFSA; also an adaptive operator is introduced to enhance the adaptive of step. Besides, a self-active inertia weight factor is introduced to improve the convergence. The IAFSA is applied to the path planning problem of mobile robot which installed ROS. Five wellknown benchmark functions are used to evaluate the performance of IAFSA. Simulation shows that the convergence speed of IAFSA is more efficient, the local search ability is enhanced, and the optimal result is more accurate. The performance of IAFSA is better than standard AFSA. In order to further analyze the superiority and effectiveness of the IAFSA in robot path planning, the IAFSA is compared experimentally with the standard AFSA, $A^{*}$ algorithm, and HAAFSA, both in self-created environment and real environment. Experiment results show that IAFSA can adapt to different environment in the planned trajectories with both satisfactory accuracy and stability. The optimal ability is better and the route, which the robot walked, is the shortest. The space complexity is reduced and the path is smoother.

In paper "Method of Quantitative Analysis for Multirobot Cooperative Hunting Behaviors," S. Yong et al. have investigated the hunting behaviors of mobile robots. The multirobot system can be adequately described based on the phase space information, and the dynamic system states can be forecast based on this information. The eigenvalues of the attractor are calculated including the maximum Lyapunov exponent and correlation dimension. The robot collective behavior is described and analyzed quantitatively based on the eigenvalues. The critical factor that affects the interaction of robots is investigated based on quantified parameters. The analysis results can be used to improve the understanding of robot interaction mechanisms.

In paper "Exact and Heuristic Algorithms for Routing AGV on Path with Precedence Constraints," L. Xu et al. considered the TSPP-PC, which is a variety of TSP. Though TSP is widely studied, whether or not TSPP-PC is polynomialtime solvable remains an open question in the literature. This paper provides a positive answer for this problem when number of precedence $k$ is part of the input size and has developed efficient heuristic for the problem with arbitrarily large $k$.

\section{Acknowledgments}

This special issue represents an exciting and insightful snapshot of the multirobot path planning research. As the Lead Guest Editor of this special issue, I would like to express our sincere gratitude to four coeditors for helping me to undertake this project with a wonderful accomplishment.
I would also like to thank interested authors who have submitted their papers to the special issue.

Jason $G u$

Xin $\mathrm{Ma}$

$\mathrm{Fei} \mathrm{Liu}$

Ying Wang

Hongliang Ren 


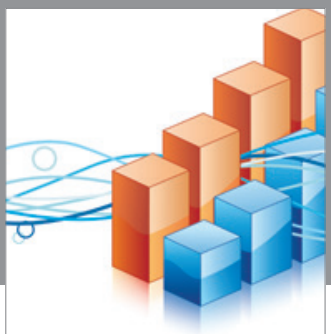

Advances in

Operations Research

vatem alat4

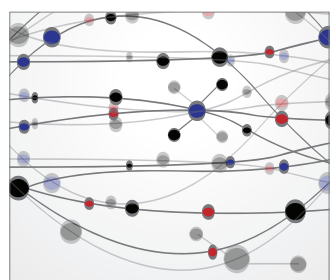

\section{The Scientific} World Journal
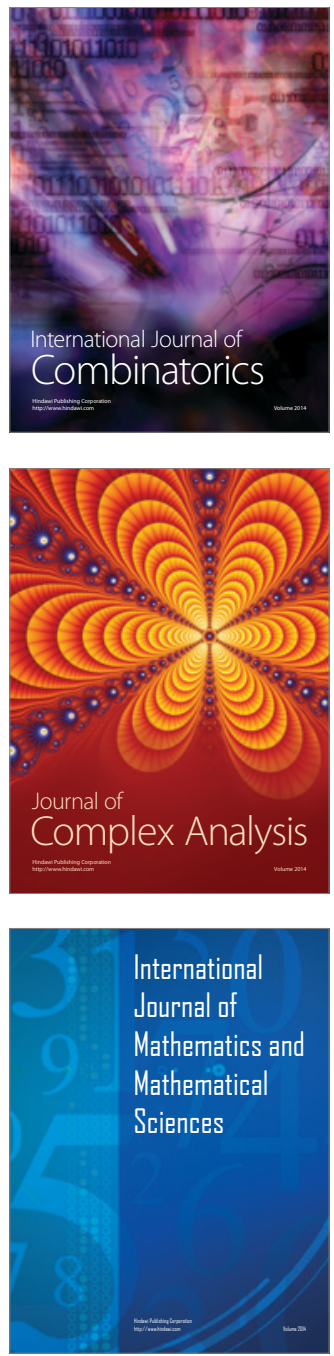
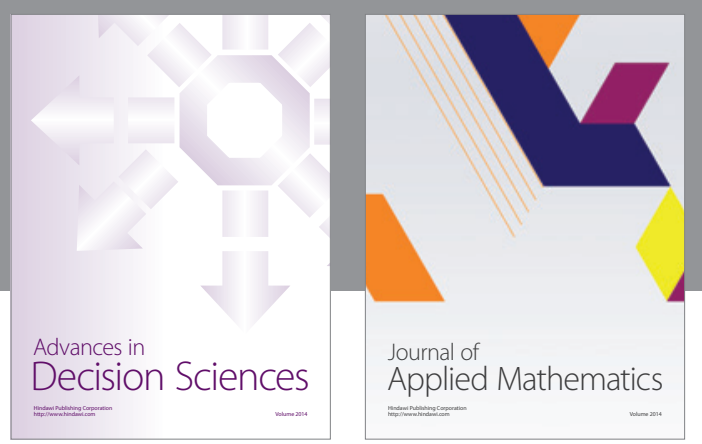

Algebra

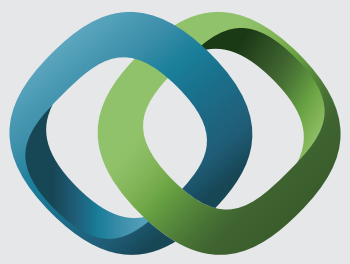

\section{Hindawi}

Submit your manuscripts at

https://www.hindawi.com
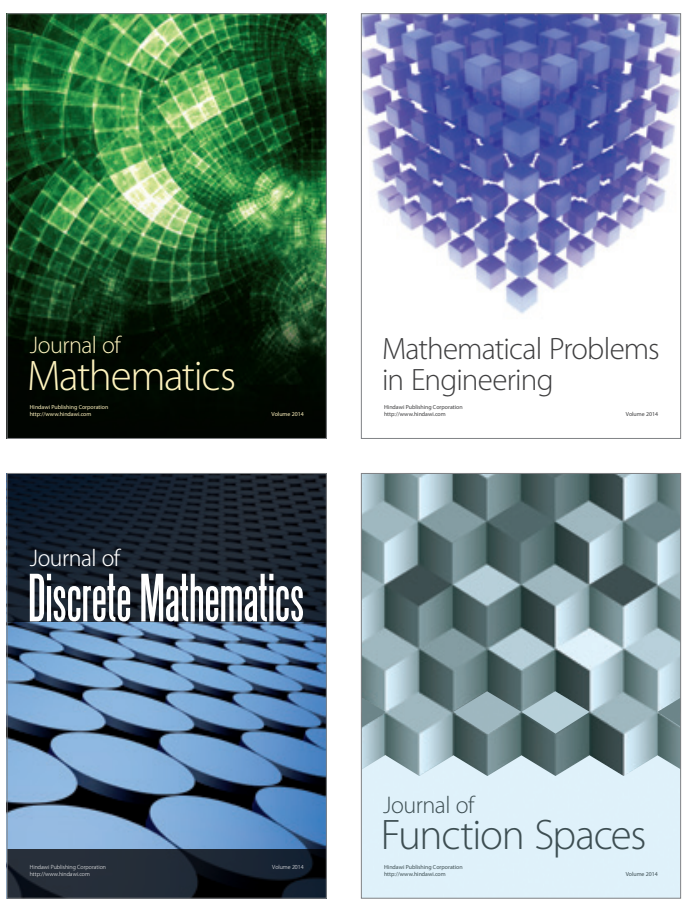

Mathematical Problems in Engineering
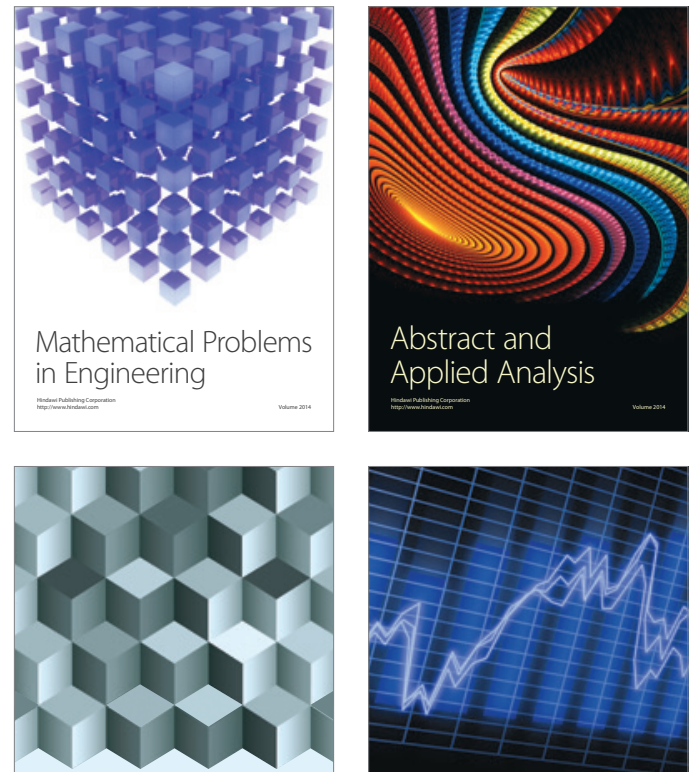

Journal of

Function Spaces

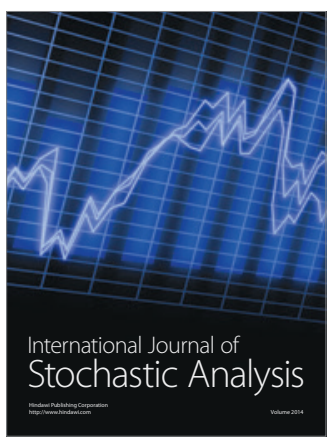

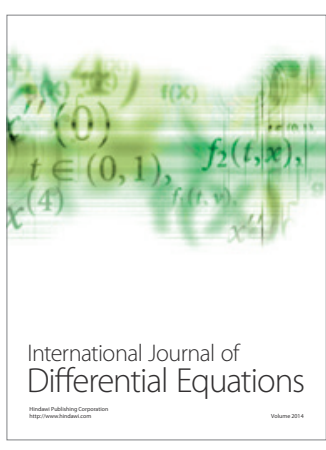
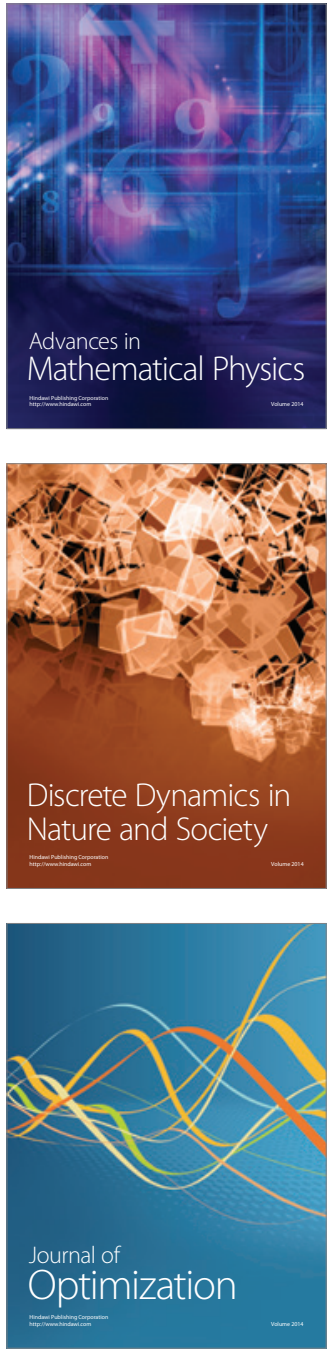